\title{
Strömgren uvby photometry of the open clusters NGC 6192 and NGC $6451^{\star}$
}

\author{
E. Paunzen ${ }^{1,2}$, H. M. Maitzen ${ }^{1}$, K. D. Rakos ${ }^{1}$, and J. Schombert ${ }^{3}$ \\ 1 Institut für Astronomie der Universität Wien, Türkenschanzstr. 17, 1180 Wien, Austria \\ 2 Zentraler Informatikdienst der Universität Wien, Universitätsstr. 7, 1010 Wien, Austria \\ 3 Department of Physics, University of Oregon, Eugene, OR 97403, USA
}

Received 19 November 2002/ Accepted 6 March 2003

\begin{abstract}
We have investigated the two open clusters NGC 6192 and NGC 6451 for which widely different reddening values and thus ages and distances via Strömgren $u v b y$ photometry can be found in the literature. Our measurements allow us to disentangle the apparent discrepancies in the literature and to derive new accurate values. From appropriate calibrations we find that the overall abundance for NGC 6192 is about solar whereas a subsolar value for NGC 6451 was estimated. From two previous reported photometrically candidate CP stars, one within NGC 6192 shows Strömgren indices typical for a B8 Si star whereas the one in NGC 6451 is most probably a foreground G-type star.
\end{abstract}

Key words. stars: chemically peculiar - stars: early-type - techniques: photometric - open clusters and associations: general

\section{Introduction}

The two open clusters NGC 6192 and NGC 6451 are good examples of how different estimates of the interstellar reddening influence the derived distances and ages. For NGC 6451, Svolopoulos (1966) lists a reddening $E(B-V)=0.08 \mathrm{mag}$ whereas Kjeldsen \& Frandsen (1991) give $E(B-V)=0.70 \mathrm{mag}$ as the best value resulting in a difference of $560 \mathrm{pc}$ versus $2100 \mathrm{pc}$ and $5 \mathrm{Gyr}$ versus $0.2 \mathrm{Gyr}$, respectively. The situation for NGC 6192 is almost identical.

We have observed these two open clusters within the Strömgren $u v b y$ system in order to derive the reddening as well as the overall metallicity. We have not tried to independently estimate the ages since no reliable isochrones within the Strömgren uvby system have been published so far. Since no $\beta$ measurements were made, the calibration of effective temperatures is not possible on solid grounds. However, ages have been derived in the literature for both clusters depending on the individual reddening values (Kjeldsen \& Frandsen 1991).

Paunzen \& Maitzen (2002) found one photometric CP candidate in each program cluster via $\Delta a$ photometry (Maitzen 1976). We have investigated the Strömgren indices for these two objects in order to clarify their nature.

Send offprint requests to: E. Paunzen,

e-mail: Ernst.Paunzen@univie.ac.at

$\star$ Based on observations at the Cerro Tololo Inter-American Observatory which is operated by the Association of Universities for Research in Astronomy Inc. (AURA), under a cooperative agreement with the National Science Foundation as part of the National Optical Astronomy Observatories.

\section{Observations and reduction}

Observations of the two open clusters and several standard stars were performed with the $150 \mathrm{~cm}$ telescope at the Cerro Tololo Inter-American Observatory. With a focus of f/7.5 and a SITe $2 \mathrm{k} \mathrm{CCD}\left(0.44^{\prime \prime} /\right.$ pixel), a field of view of about $15^{\prime}$ was achieved.

We used a standard Strömgren uvby filter set. The observing $\log$ is listed in Table 1. In total, 26 frames for the two clusters in four filters were observed and used for further analysis.

The basic reductions (bias-subtraction, dark-correction, flat-fielding) were carried out within standard IRAF routines. For all frames we applied a point-spread-function-fitting within the IRAF task DAOPHOT (Stetson 1987). Photometry of each frame was performed separately and the measurements (corrected for the extinction) were then averaged and weighted by their individual photometric error.

The finding charts of our open clusters are shown in Fig. 1. The size of the symbols (by area) is inversely proportional to the apparent visual magnitudes of the objects in the sense that larger symbols denote brighter objects.

The complete tables with all photometric data for the two cluster stars are available in electronic form at the CDS via anonymous ftp to cdsarc.u-strasbg. $f r(130.79 .128 .5)$ or http://cdsweb.u-strasbg. fr/cgi-bin/qcat?]/A+A/403/937 and can be requested from the first author. These tables include the cross identification of objects from the literature, the observed $(b-y), m_{1}$ and $c_{1}$ values with their corresponding errors, $V$ magnitudes, as well as the $(B-V)$ and $(U-B)$ values from the literature. 


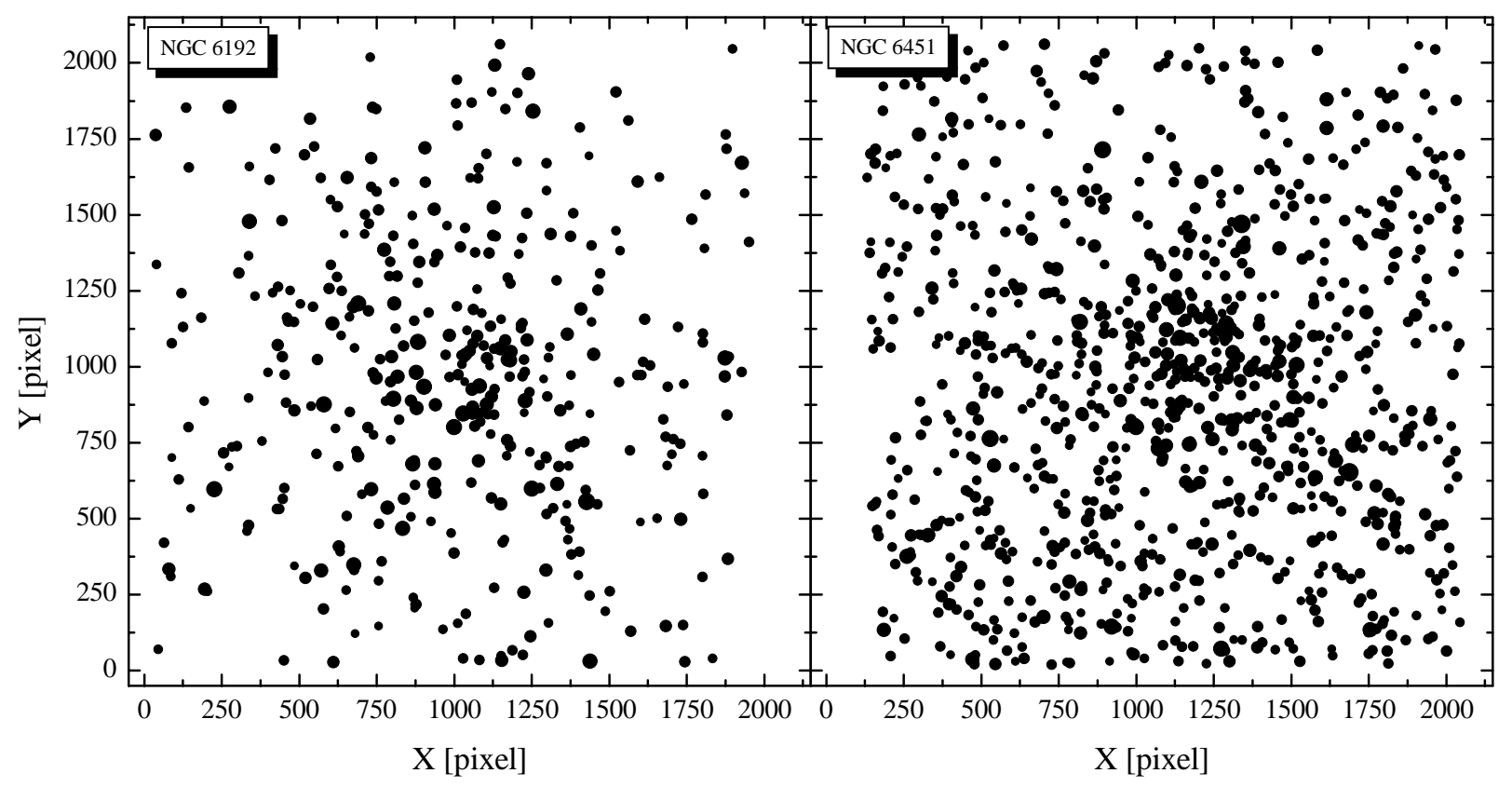

Fig. 1. Finding charts for the program clusters. North is to the left and west is upwards; 1 pixel $=0.43^{\prime \prime}$. The sizes (by area) of the open circles are inversely proportional to the $V$-magnitudes taken from Tables 2 and 3 in the sense that larger open circles denote brighter objects.

Table 1. Observing log.

\begin{tabular}{lccccc}
\hline \hline Cluster & Night & $\#_{u}$ & $\#_{v}$ & $\#_{b}$ & $\#_{y}$ \\
\hline NGC 6192 & $02 / 03.09 .2002$ & 2 & 2 & 2 & 2 \\
NGC 6451 & $02 / 03.09 .2002$ & 2 & 2 & 2 & 2 \\
& $03 / 04.09 .2002$ & 2 & 3 & 3 & 2 \\
\hline
\end{tabular}

\section{Calibration into the standard photometric system}

A very important point is the calibration of the observed photometric values to standard ones. For the determination of the extinction of the individual filters, three spectrophotometric standard stars LTT 377, LTT 1020 and LTT 9239 were observed. Spectrophotometric as well as broadband Johnson UBVRI photometry for these objects can be found in Hamuy et al. (1992, 1994) and Landolt (1992). Unfortunately, no Strömgren uvby measurements for these objects are available. However, we have used the spectrophotometric measurements and the standard Strömgren $u v b y$ transmission curves (Crawford \& Barnes 1970) to calculate "synthetic" photometric indices for these three standard stars. The small range of the color indices (e.g. $<0.1 \mathrm{mag}$ for $b-y$ ) makes any reliable calibration for the whole relevant color range (more than $1.2 \mathrm{mag}$; see Fig. 3) very problematic. However, we used the calculated photometric indices to derive absolute fluxes using the calibration of Gray (1998). He lists absolute flux calibrations for the Strömgren $u v b y$ bands on the basis of data from Vega. The data for our three standard stars are intrinsically consistent and a comparison with the the results listed for the Johnson UBVRI by Hamuy et al. (1992, 1994) and Landolt (1992) shows an excellent agreement.

For the determination of the extinction coefficients we closely follow the approach described in Sung \& Bessell (2000). For this purpose we used the measurements of the three standard stars at airmasses from 1.0 to 2.0 on the two different nights. The coefficients were found to be $0.48,0.31,0.18$ and 0.11 for $u, v, b$ and $y$, respectively.

For the calibration of the instrumental $b-y, m_{1}$ and $c_{1}$ values we used the following procedure. The observed CCD photometric $B-V$ values for NGC 6192 (King 1987; Kjeldsen \& Frandsen 1991) and NGC 6451 (Kjeldsen \& Frandsen 1991) were transformed into $b-y$ magnitudes using the correlations listed in Caldwell et al. (1993). These values served as a standard system for the calibration of our instrumental values. Figure 2 shows the $(b-y)_{\text {stand }}$ versus $(b-y)_{\text {inst }}$ diagram with the available data for both open clusters. The line fits the data of the two clusters very well. Taking the calibrated $b-y$ magnitudes, $\left[m_{1}\right]$ and $\left[c_{1}\right]$ indices were calculated using the absolute fluxes derived for the three standard stars as described above. Since these indices are not sensitive to reddening (Crawford \& Mander 1966), the offsets of the instrumental $m_{1}$ and $c_{1}$ can be estimated. The $y$ magnitudes were directly converted into standard Johnson $V$ ones using the data of King (1987) and Kjeldsen \& Frandsen (1991).

We find the following transformation for the instrumental magnitudes:

$$
\begin{aligned}
& V=-3.02(4)+0.997(2) \cdot y_{i} \\
& b-y=-0.103(9)+0.921(14) \cdot(b-y)_{i} \\
& m_{1}=-0.723(9) \cdot\left(m_{1}\right)_{i} \\
& c_{1}=+0.146(8) \cdot\left(c_{1}\right)_{i}
\end{aligned}
$$

These equations hold for the data of both open clusters.

\section{Program cluster}

In the following two sections we will summarize the already published information about NGC 6192 and NGC 6451. 


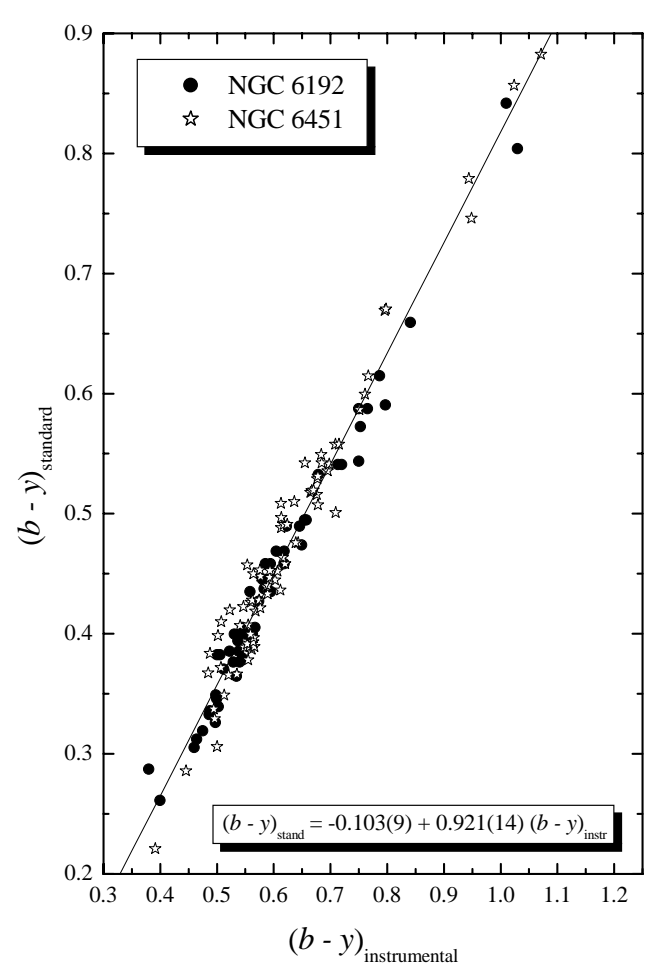

Fig. 2. $(b-y)_{\text {standard }}$ versus $(b-y)_{\text {instrumental }}$ diagram for our program clusters.

\subsection{NGC 6192}

Kilambi \& Fitzgerald (1983; photographic photometry) and King (1987; CCD photometry) reported a rather old age $(1300 \mathrm{Myr})$ with a reddening of $E(B-V)=0.26 \mathrm{mag}$ and a distance of about $1000 \mathrm{pc}$ from the Sun. Kjeldsen \& Frandsen (1991; CCD photometry) on the other hand list a reddening of $E(B-V)=0.68 \mathrm{mag}$, an age of $89 \mathrm{Myr}$ and a distance of about $1700 \mathrm{pc}$ from the Sun. The sources of CCD photometry agree very well and have been used for our analysis.

Paunzen \& Maitzen (2002) analysed all published photometric data and found that the age, reddening and distance modulus from Kjeldsen \& Frandsen (1991) give the best fit. They also reported the detection of one photometric CP candidate (No.54 according to King 1987; No.86, Kjeldsen \& Frandsen 1991) with $\Delta a=+0.030 \mathrm{mag}$. From their photometry a spectral type of $\mathrm{B} 8 \mathrm{Si}$ and an absolute magnitude of $M_{V}=+0.0(2)$ mag was concluded.

\subsection{NGC 6451}

Paunzen \& Maitzen (2002) investigated the discrepancies in the data published by Piatti et al. (1998) and Kjeldsen \& Frandsen (1991) for this open cluster. Piatti et al. (1998) lists $E(B-V)=0.08$ mag whereas Kjeldsen \& Frandsen (1991) give $E(B-V)=0.70 \mathrm{mag}$ as the best value. This results in a difference of $560 \mathrm{pc}$ versus $2100 \mathrm{pc}$ and $5 \mathrm{Gyr}$ versus $0.2 \mathrm{Gyr}$, respectively. Since Piatti et al. (1998) calibrated their isochrones using the data for NGC 6451, we decided to observe this open cluster via Strömgren uvby photometry.
Table 2. Summary of results; the ages were taken from the literature. In parenthesis are the errors in the final digits of the corresponding quantity.

\begin{tabular}{lcc}
\hline \hline Name & NGC 6192 & NGC 6451 \\
& C1636-432 & C1747-302 \\
\hline$l / b$ & $341 /+2$ & $360 /-2$ \\
Tr-type & I 2 r & I 2 r \\
$E(b-y)$ & $0.40(5)$ & $0.40(5)$ \\
$V_{0}-M_{V}$ & $11.40(10)$ & $12.15(10)$ \\
$d[\mathrm{pc}]$ & $1900(100)$ & $2690(100)$ \\
{$[\mathrm{Fe} / \mathrm{H}]$} & $-0.10(9)$ & $-0.34(6)$ \\
$\log t$ & 7.95 & 8.30 \\
$n($ obj $)$ & 374 & 912 \\
$n$ (frames) & 18 & 12 \\
\hline
\end{tabular}

Paunzen \& Maitzen (2002) concluded that the $V$ magnitudes given by Piatti et al. (1998) are on average one magnitude too bright and thus the reddening, age and distance listed within this reference are probably incorrect. They refitted the data of Piatti et al. (1998) and found $E(B-V)=0.60 \mathrm{mag}$, $\left(V-M_{V}\right)=13.90$ and an age of about $0.4 \mathrm{Gyr}$ which is consistent with the results of Kjeldsen \& Frandsen (1991).

Furthermore, Paunzen \& Maitzen (2002) found one photometric CP candidate within the boundaries of NGC 6451.

\section{Results}

First we estimated the reddening of NGC 6192 and NGC 6451. Taking the standard relation of Philip \& Egret (1980), the observed magnitudes were shifted accordingly. Figure 3 shows the $M_{V}$ versus $(b-y)_{0}$ diagrams. The reddening of both program clusters was found to be $E(b-y)=0.40(5) \mathrm{mag}$. This is well in line with the estimates of Kjeldsen \& Frandsen (1991) and clearly contradicts the values listed by King (1987; NGC 6192) and Piatti et al. (1998; NGC 6451). For the distance modulus we find $\left(V_{0}-M_{V}\right)=11.40(10)$ and $12.15(10)$ resulting in a distance of 1900(100) as well as 2690(100)pc for NGC 6192 and NGC 6451, respectively. Our results agree within $1 \sigma$ (NGC 6192) and $3 \sigma$ (NGC 6451) with those of Kjeldsen \& Frandsen (1991). Several red giants were clearly visible for NGC 6192 whereas several bright $\left(M_{V}>-2.5 \mathrm{mag}\right)$ non-members for NGC 6451 were detected. We have not estimated the ages of both open clusters since no isochrones for the Strömgren uvby system are available. The only way would be the transformation of the $(b-y)_{0}$ magnitudes into effective temperature which is strongly dependent on (unavailable) $\beta$ measurements. However, the ages listed in the literature are compatible with those derived from a comparison with the isochrones given in Schulz et al. (2002). Table 2 lists all results deduced from our analysis.

The $M_{V}$ versus $(b-y)_{0}$ diagram for NGC 6451 shows a clustering of objects parallel to the main sequence at $0.4 \leq$ $(b-y)_{0} \leq 0.7$ and $+1.0 \leq M_{V} \leq-0.5$. We have investigated the location of these objects with respect to the field of NGC 6451 (Fig. 4). The placement of these stars is randomly distributed over the whole observed area ruling out a possible 


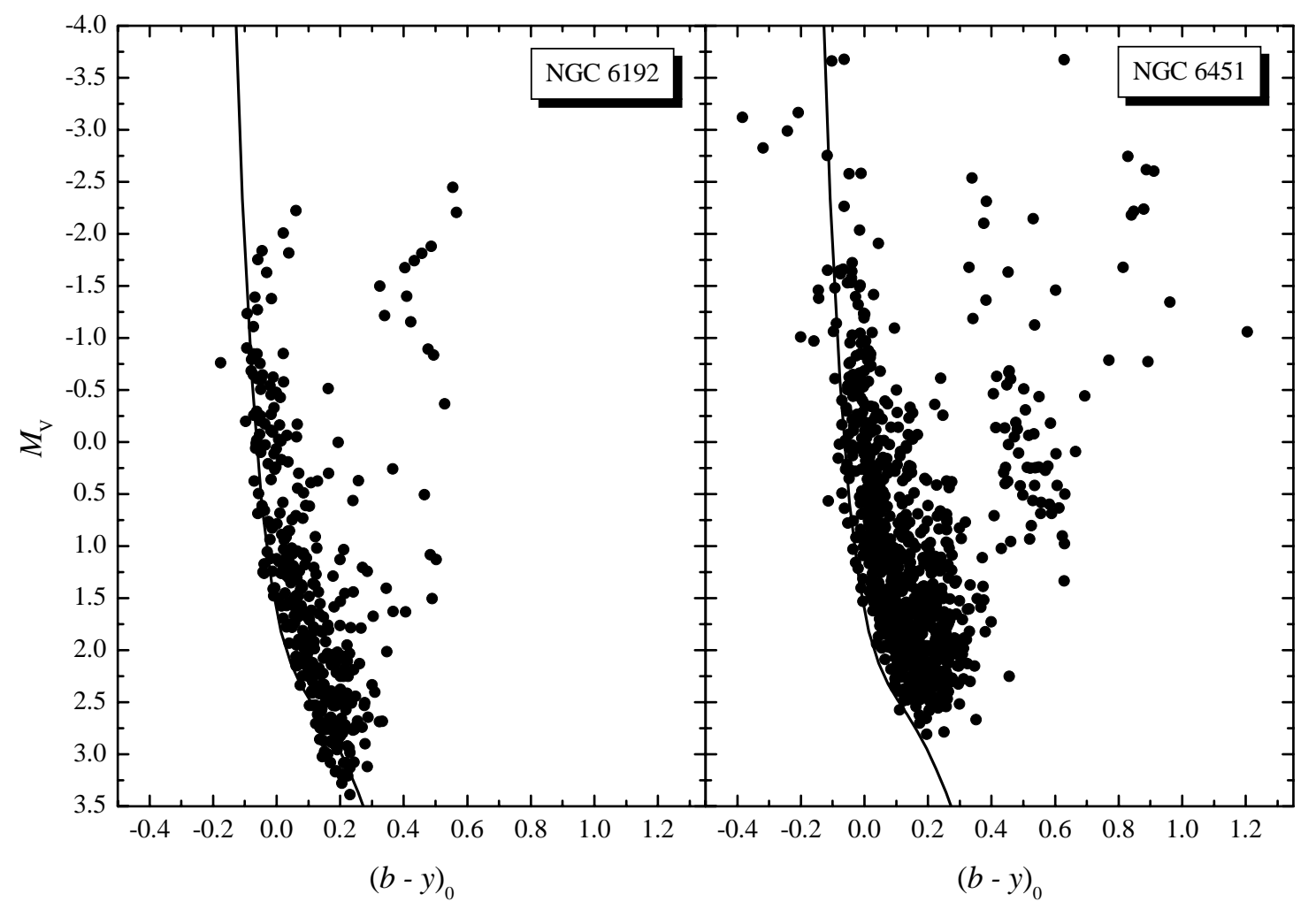

Fig. 3. $M_{V}$ versus $(b-y)_{0}$ diagrams for our program clusters; the standard relation is taken from Philip \& Egret (1980).

inconsistency in the reduction process as well as a second, more distant, open cluster within the vicinity of NGC 6451 . Since almost none of these objects are within the innermost boundaries of NGC 6451, we believe that the clustering found is a manifestation of the more distant field population.

As a next step, the overall metallicities for both open clusters was estimated. For this purpose the calibration of Nissen (1981) for stars cooler than F0 was taken. Since no $\beta$ measurements are available we used the standard relations of Crawford (1975) and Olsen (1984) to estimate them for the relevant temperature domain. Such a procedure has to be used with caution. Applying this method results in $[\mathrm{Fe} / \mathrm{H}]=-0.10(9)$ and -0.34(8) for NGC 6192 and NGC 6451, respectively. The errors in brackets are the errors of the means. This seems to indicate that the metallicity for NGC 6451 is slightly subsolar. Another method to calibrate metallicities for red giants is listed by Hilker (2000). This calibration is valid for objects with $0.5<(b-y)_{0}<1.0 \mathrm{mag}$. This fact together with the apparent uncertainty of the membership of objects for NGC 6451 seriously limits the significance of the derived values. Only two and five objects remain for NGC 6192 and NGC 6451, respectively, which results in errors as large as \pm 0.5 dex.

The investigation of the photometric candidate CP star in NGC 6192 (Paunzen \& Maitzen 2002) further strengthens its chemically peculiar nature. We find $(b-y)_{0}=-0.026$, $\left[m_{1}\right]=0.202,\left[c_{1}\right]=0.738$ and $M_{V}=0.21$ mag which are typical for a B8 Si star (Cameron 1966).

The values for the apparent peculiar object in NGC 6451 are typical for an unreddened G-type star (Crawford \& Mander 1966) and rule out a chemical peculiarity: $(b-y)=0.651$,

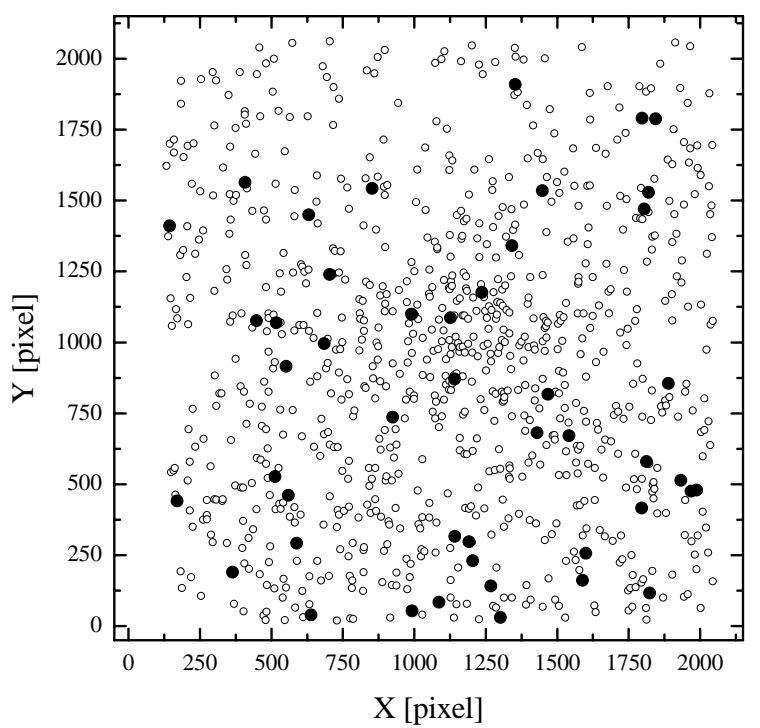

Fig. 4. The distribution of apparent members (open circles) of NGC 6451 and the "shifted population" as described in Sect. 5.

$\left[m_{1}\right]=0.467,\left[c_{1}\right]=0.233$ and $M_{V}=3.85 \mathrm{mag}$. This case shows the importance of further photometric or spectroscopic investigations of candidate $\mathrm{CP}$ stars.

\section{Conclusions}

We have presented Strömgren $u v b y$ photometry for the young open clusters NGC 6192 (374 objects) and NGC 6451 (912 objects). For both open clusters, widely different reddening 
values and thus distances and ages can be found in the literature. From our photometry we were able resolve these discrepancies and derive new accurate values. The overall metallicities were found to be about solar for NGC 6192 as well as slightly subsolar for NGC 6451 using the appropriate calibrations from the literature.

The chemically peculiar nature for one object of NGC 6192 was proven whereas one photometric $\mathrm{CP}$ candidate within NGC 6451 has to be rejected.

Acknowledgements. EP acknowledges partial support by the Fonds zur Förderung der wissenschaftlichen Forschung, project P14984. Use was made of the SIMBAD database, operated at CDS, Strasbourg, France and the WEBDA database, operated at the Institute of Astronomy of the University of Lausanne.

\section{References}

Caldwell, J. A. R., Cousins, A. W. J., Ahlers, C. C., van Wamelen, P., \& Maritz, E. J. 1993, SAAO Circulars, No. 15

Cameron, R. C. 1966, Georgetown Obs. Monogram, 21

Crawford, D. L. 1975, AJ, 80, 955
Crawford, D. L., \& Barnes, J. V. 1970, AJ, 75, 978

Crawford, D. L., \& Mander, J. 1966, AJ, 71, 114

Gray, R. O. 1998, AJ, 116, 482

Hamuy, M., Walker, A. R., Suntzeff, N. B., et al. 1992, PASP, 104, 533

Hamuy, M., Suntzeff, N. B., Heathcote, S. R., et al. 1994, PASP, 106, 566

Hilker, M. 2000, A\&A, 355, 994

Kilambi, G. C., \& Fitzgerald, M. P. 1983, Bull. Astr. Soc. India, 11, 226

King, D. J. 1987, Observatory, 107, 107

Kjeldsen, H., \& Frandsen, S. 1991, A\&AS, 87, 119

Landolt, A. U. 1992, AJ, 104, 372

Maitzen, H. M. 1976, A\&A, 51, 223

Nissen, P. E. 1981, A\&A, 97, 145

Olsen, E. H. 1984, A\&AS, 57, 443

Paunzen, E., \& Maitzen, H. M. 2002, A\&A, 385, 867

Philip, A. G., Davis, \& Egret, D. 1980, A\&AS, 40, 199

Piatti, A. E., Clariá, J. J., \& Bica, E. 1998, ApJS, 116, 263

Schulz, J., Fritze-v. Alvensleben, U., Möller, C. S., \& Fricke, K. J. 2002, A\&A, 392, 1

Stetson, P. B. 1987, PASP, 99, 191

Sung, H., \& Bessell, M. S. 2000, PASA, 17, 244

Svolopoulos, S. N. 1966, Z. Astrophys., 64, 67 